\title{
Itinéraires
}

Itinéraires Littérature, textes, cultures

\section{Bibliographie sélective}

\section{(2) OpenEdition \\ 1 Journals}

Édition électronique

URL : http://journals.openedition.org/itineraires/639

DOI : 10.4000/itineraires.639

ISSN : 2427-920X

Éditeur

Pléiade

\section{Édition imprimée}

Date de publication : 1 mars 2014

Pagination : 209-215

ISBN : 978-2-343-02712-8

ISSN : 2100-1340

Référence électronique

«Bibliographie sélective », Itinéraires [En ligne], 2013-2 | 2014, mis en ligne le 01 novembre 2013, consulté le 15 septembre 2020. URL : http://journals.openedition.org/itineraires/639

\section{(c) (i) (9)}

Itinéraires est mis à disposition selon les termes de la licence Creative Commons Attribution - Pas d'Utilisation Commerciale - Pas de Modification 4.0 International. 


\section{Bibliographie sélective}

\section{Essais}

Abramovici Jean-Christophe, Encre de sang. Sade écrivain, Paris, Classiques Garnier, 2013.

Apollinaire Guillaume, L'Euvre du Marquis de Sade, Paris, Bibliothèque des curieux, 1912.

BADINTER Élisabeth, Émilie, Émilie. L'ambition féminine au XVIII siècle, Paris, Flammarion, 1983.

BosQuet Marie-Françoise, Images du féminin dans les utopies françaises classiques, Oxford, Voltaire Foundation, 2007.

Barthes Roland, Sade, Fourier, Loyola, Paris, Seuil, 1971.

Beauvorr Simone de, Faut-il brûler Sade?, Paris, Gallimard, 1955.

—, Le Deuxième Sexe, Paris, Gallimard, 1949, rééd. 1976.

BRouARDS-ARENDS Isabelle, Vie et images maternelles dans la littérature française du dix-huitième siècle, Oxford, Voltaire Foundation, coll. «SVEC », 1991.

BUtLer Judith, Trouble dans le genre. Le féminisme et la subversion de l'identité [1990], Paris, La Découverte, 2006.

CArter Angela, La Femme sadienne, Paris, Henri Veyrier, 1979.

CAndler Hayes Julie, Identity and Ideology: Diderot, Sade, and the serious genre, Amsterdam - Philadelphie, John Benjamins, 1991.

Coudreuse Anne, Le Goût des larmes au XVIII siècle, Paris, PUF, 1999.

- Le Refus du pathos au XVIII siècle, Paris, Champion, 2001.

Cusset Catherine (dir.), Libertinage and Modernity, New Haven - Londres, Yale University Press, coll. «Yale french studies », nº 94, 1998.

Delon Michel et Seth Catriona (dir.), Sade en toutes lettres. Autour d'Aline et Valcour, Paris, Desjonquères, 2004.

Delon Michel, L'Idée d'énergie au tournant des Lumières (1770-1820), Paris, PUF, 1988.

- Le Savoir-vivre libertin, Paris, Hachette, 2000. 
Denby David, Sentimental Narrative and the Social Order in France, 1760-1820, Cambridge, Cambridge University Press, 1994.

Didier Béatrice, Sade, une écriture du désir, Paris, Denoël, 1976.

DiJK Suzan van et Strien-Chardonneau Madeleine van (dir.), Féminités et masculinités dans le texte narratif avant 1800. La question du " gender », Louvain, Peeters, 2002.

DurAND Béatrice, Le Paradoxe du bon maître. Essai sur l'autorité dans la fiction pédagogique des Lumières, Paris, L'Harmattan, 1999.

Dworkin Andrea, Pornography. Men Possessing Women, New York, Penguin, 1979.

Edminston William F., Sade: Queer Theorist, Oxford, Voltaire Foundation, coll. « SVEC », 2013.

FABRE Jean, Idées sur le roman, de Madame de Lafayette au Marquis de Sade, Paris, Klincksieck, 1979.

FAuchery Pierre, La Destinée féminine dans le roman européen du dixhuitième siècle : 1713-1807, essai de gynécomythie romanesque, Paris, Armand Colin, 1972.

FEND Mechthild, Les Limites de la masculinité. L'androgyne dans l'art et la théorie de l'art en France (1750-1830), Paris, La Découverte, 2011.

FraPPIER-MAzUr Lucienne, Sade et l'écriture de l'orgie : pouvoir et parodie dans l'Histoire de Juliette, Paris, Nathan, 1991.

Gallop Jane, Intersections: A Reading of Sade with Bataille, Blanchot and Klossowski, Lincoln - Londres, University of Nebraska Press, 1981.

Galster Ingrid (dir.), Le Deuxième Sexe de Simone de Beauvoir, Paris, Presses de 1'Université Paris-Sorbonne, 2004.

GENAND Stéphanie, Le Libertinage et l'Histoire : politique de la séduction à la fin de l'Ancien Régime, Oxford, Voltaire Foundation, coll. « SVEC», 2005.

Hesse Carla, The Other Enlightenment. How French women became modern, Princeton, Princeton University Press, 2001.

Huston Nancy, Mosaïque de la pornographie, Paris, Denoël, 1982.

JeAngène ViLmer Jean-Baptiste, La Religion de Sade, Paris, Les Éditions de l'Atelier, 2008.

KLossowski Pierre, Sade mon prochain, Paris, Seuil, 1947.

Kozul Mladen, Le Corps dans le monde. Récits et espaces sadiens, Louvain, Peeters, 2005.

LaCan Jacques, Éthique de la psychanalyse, Séminaire VII, Paris, Seuil, 1986.

- Écrits, Paris, Seuil, 1966.

- Encore, Séminaire XX, Paris, Seuil, 1975. 
Le Brun Annie, Sade, aller et détours, Paris, Plon, 1989.

— «Pourquoi Juliette est-elle une femme ?», dans On n'enchaîne pas les volcans, Paris, Gallimard, 2006, p. 127-157.

- Soudain un bloc d'abîme, Sade : introduction aux ouvres complètes, Paris, Pauvert, 1986.

- Les Châteaux de la subversion, Paris, Gallimard, 1982.

LotTERIE Florence, Le Genre des Lumières. Femme et philosophe au XVIII siècle, Paris, Classiques Garnier, 2013.

Lugan Anne-Marie, Les Châteaux d'Eros ou les Infortunes du sexe des femmes, Paris, Maspero, 1980.

Martin Christophe, Espaces du féminin dans le roman français du dixhuitième siècle, Oxford, Voltaire Foundation, coll. « SVEC », 2004.

- "Éducations négatives ». Fiction d'expérimentation pédagogique au dix-huitième siècle, Paris, Classiques Garnier, 2010.

Marty Éric, Pourquoi le Xx siècle a-t-il pris Sade au sérieux, Paris, Seuil, 2011.

Mathieu Nicole-Claude, L'Anatomie politique. Catégorisations et idéologies du sexe, Paris, Côté-femmes, 1991.

Miller Nancy, French Dressing. Women, Men and Ancien Régime Fiction, New York - Londres, Routledge, 1995.

Paschoud Adrien et Wenger Alexandre (dir.), Sade : sciences, savoirs et invention romanesque, Paris, Hermann, 2012.

Phillips John, How to read Sade, Londres, Granta, 2005.

Plessis Gray Francine du, At home with the Marquis de Sade, Londres, Chatto \& Windus, 1999.

Roger Philippe, Sade, La Philosophie dans le pressoir, Paris, Grasset, 1976.

SEmInet Philippe, Sade in his own name: an analysis of Les Crimes de l'amour, New York, Peter Lang, 2003.

SontAG Susan, « The pornographic imagination », dans Styles of Radical Will [1966], Londres, Vintage, 1994.

- L'Cuvre parle [1966], Paris, Seuil, 1968.

Thomas Chantal, Sade, l'œil de la lettre, Paris, Payot, 1978.

- Sade, la dissertation et l'orgie, Paris, Payot, 1978.

Trouille Mary, Wife-abuse in eighteenth-century France, Oxford, Voltaire Foundation, coll. « SVEC », 2009.

Warman Caroline, Sade: from materialism to pornography, Oxford, Voltaire Foundation, coll. « SVEC », 2002.

Wynn Thomas, Sade's theatre: pleasure, vision, masochism, Oxford, Voltaire Foundation, coll. « SVEC », 2007. 
GARAND Caroline et Wynn Thomas (dir.), « Sade au théâtre : la scène et l'obscène ", L'Annuaire théâtral : revue québécoise d'études théâtrales, no 41, 2007.

Zajac Marta, The Feminine of Difference, Gilles Deleuze, Hélène Cixous and Contemporary Critique of the Marquis de Sade, Francfort, Peter Lang, 2002.

\section{Articles}

Abramovici Jean-Christophe, «"Faut-il brûler Sade ?” Rigueur de l'empathie », Cahiers de l'Herne, n 100, 2012, p. 316-320.

ANDRÉ Arlette, « Sade et l'éthique de l'apathie », dans Mélanges littéraires, François Germain, Dijon, Faculté de lettres et philosophie, 1979, p. 95-104.

BAuer-Funke Cerstin, «Aline et Léonore ou les effets de la violence : violence et progrès dans Aline et Valcour ou Le roman philosophique du marquis de Sade », dans Valérie Cossy et Deidre Dawson (dir.), Progrès et violence au XVIII siècle, Paris, Champion, 2001, p. 167-186.

BenreKassa Georges, « Loi naturelle et loi civile : 1'idéologie des Lumières et la prohibition de l'inceste », SVEC, no 87, 1972, p. 115-144.

Bonnet Jean-Claude, "Sade historien », dans Michel Camus et Philippe Roger (dir.), Sade, écrire la crise, Paris, Belfond, 1983, p. 133-148.

Butler Judith, « Beauvoir on Sade: making sexuality into an ethic », dans Claudia Card (dir.), The Cambridge Companion to Simone de Beauvoir, 2003 , trad. française L'Homme et la société, n 150-151, oct. 2003-mars 2004, p. 69-97.

Cerruti Giorgio, « Le paradoxe sur le comédien et le paradoxe sur le libertin, Diderot et Sade », Revue des sciences humaines, n 37, 1972, p. 5974.

Chatelet Noëlle, « Juline », Obliques, nº 12-13, 1977, p. 61-63.

Claude Catherine, « Une lecture de femme », Europe, n 522, 1972, p. 64-70.

Coudreuse Anne, « Justine ou les bonheurs de la curiosité », dans Nicole Jacques-Lefèvre et Sophie Houdard (dir.), Curiosité et libido sciendi de la Renaissance, Fontenay-aux-Roses, ENS éditions, coll. « Theoria », 1998, t. II, p. 393-422.

—, « Éthique et politique dans le théâtre de Sade », dans Norbert Sclippa (dir.), Lire Sade, Paris, L'Harmattan, 2004, p. 187-207.

—, « Porc frais de mes pensées... : délire et destination dans les Lettres à sa femme et le Journal de Charenton de Sade », Épistolaire, no 32, 2006, p. 91-100.

CRYLE Peter, «État présent de la critique sadienne », Dix-huitième siècle, $n^{\circ} 31,1999$, p. 507-524. 
Cusset Catherine, « La passion selon Juliette », L'Infini, no 31, 1990, p. 405-406.

—, «Sade, Machiavel et Néron : de la théorie politique à l'imaginaire libertin », Dix-huitième siècle, n 22 , 1990, p. 401-412.

Dobay Rifelu Carol de, «Cendrillon and the Ogre: Women in Fairy Tales and Sade », Romanic Review, no 81, 1990, p. 11-24.

Delon Michel, « Dix années d'études sadiennes (1968-1978) », Dix-huitième siècle, no 11, 1979, p. 329-426.

— «Le prétexte anatomique », Dix-huitième siècle, $\mathrm{n}^{\circ}$ 12, 1980, p. 35-48.

- « Les historiennes de Silling », dans L'Histoire au XVIII siècle, La Calade, Édisud, 1980, p. 101-113.

— «Sade autobiographe : les personnages de Valcour et de Rodin », dans Mary Donaldson-Evans, Lucienne Frappier-Mazur et Gerald Prince (dir.), Autobiography, Historiography, Rhetoric. A Festschrift in honor of Frank Paul Bowman, Amsterdam, Rodopi, 1994, p. 75-86.

— " "La décharge de Saint-Fond était brillante". Éloge et critique, chez Sade, de l'ostentation sociale », dans Anne Chamayou (dir.), La Littérature et le brillant. Mélanges en l'honneur de Pierre Malandain, Arras, Artois Presses Université, 2002, p. 203-210.

— « Jeanne Laisné, héroïne sadienne », dans Paul Mironneau et Gérard Lahouati (dir.), Figures de l'histoire de France dans le théâtre au tournant des Lumières (1760-1830), Oxford, Voltaire Foundation, coll. « SVEC », 2007, p. 81-88.

— « L'obsession anale de Sade », Annales historiques de la Révolution française, $\mathrm{n}^{\circ} 361,2010$, p. 131-144.

Deprun Jean, « Sade et la philosophie biologique de son temps », dans Michel Camus et Philippe Roger (dir.), Sade, écrire la crise, Paris, Belfond, 1983, p. 189-203.

Didier Béatrice, « Juliette, femme forte de l'écriture sadienne », Obliques, $\mathrm{n}^{\circ} 14-15,1977$, p. 271-277.

— «Inceste et écriture chez Sade », Les Lettres nouvelles, n 3, 1972, p. $150-158$.

— «L'image du Moyen Âge chez Sade », La Licorne, n 6, 1982, p. 301311.

FInAS Lucienne, "L'increvable féminin dans Faxelange », dans Michel Camus et Philippe Roger (dir.), Sade, écrire la crise, Paris, Belfond, 1983, p. 15-29.

FINK Béatrice, «Ambivalence in the gynogram: Sade's utopian woman », Women in literature, vol. 7, $\mathrm{n}^{\circ} 1,1979$, p. 24-37. 
GaIllard Aurélia, «Aimer une statue : Pygmalion ou la fable de l'amour comblé », Intermédialités : histoire et théorie des arts, des lettres et des techniques, ${ }^{\circ}$ 4, 2004, p. 67-85.

GAMBACORTI Chiara, «"Ces tableaux du crime triomphant...” Écriture moralisante et perversion textuelle dans Les Crimes de l'amour de Sade », Dix-huitième siècle, no 39, 2007, p. 543-554.

Genand Stéphanie, «Entre asservissement sexuel et despotisme politique. La figure du bourreau chez Sade », Labyrinthe, no 13, 2002, p. 33-49.

— «Anthropophagie et despotisme chez Sade : l'homme est-il un animal politique ? », École thématique, ENS Lyon, 2010 [En ligne], http:// ecole-thema.ens-lyon.fr/IMG/pdf/Article_Genand-2.pdf.

Guilmette Lauren, « Reading Butler reading Beauvoir reading Sade. On ethics and Eros », Philosophy Today, 2011, p. 292-301.

Guyaux André, «Théâtre de Sade », Revue d'histoire du théâtre, $\mathrm{n}^{\circ}$ 121, 1979, p. 46-51.

KLossowski Pierre, « Justine et Juliette », Obliques, n 12-13, 1977, p. 249252.

Knapp-Tepperberg E.-M., « Warum ist der Marquis de Sade kein "feministischer" Autor? », Lendemains. Zeitschrift für Frankreichforschung, vol. 5, no 17-18, 1980, p. 125-137.

KRIEF Huguette, " Le génie féminin. Propos et contre-propos au XVIII ${ }^{e}$ siècle », dans Éliane Viennot (dir.), avec la coll. de Nicole Pellegri, Revisiter la " querelle des femmes ». Discours sur l'égalitélinégalité des sexes, de 1750 aux lendemains de la Révolution, Saint-Étienne, Publications de l'Université de Saint-Étienne, 2012, p. 61-76.

KURTz Robert, « La femme comme chienne de l'homme », Illusio, no 4-5, 2007, p. 526-536.

LaVERs Annette, "Le mythe de la femme dans la vie quotidienne », dans Ingrid Galster (dir.), Simone de Beauvoir : Le Deuxième Sexe. Le livre fondateur du féminisme moderne en situation, Paris, Champion, 2004, p. 261-280.

Link-HeER Ursula, "Was the "divine Marquis" and Advocate of Virtue? Remarks on the Paradox of the Sadian Theatre », dans Peter Wagner (dir.), Erotica and the Enlightenment, Francfort, Peter Lang, 1991, p. $70-89$.

LotTerie Florence, "Sade et les fictions du despotisme. Romans de la courtisane et fable de la "parrêsia" ", dans Éléonore Reverzy, Romuald Fonkoua et Pierre Hartmann (dir.), Les Fables du politique des Lumières à nos jours, Strasbourg, Presses universitaires de Strasbourg, 2012, p. 41-57. 
— «Hybrides philosophiques : quelques enjeux du dialogue matérialiste dans Le Reve de D'Alembert et La Philosophie dans le boudoir ", Recherches sur Diderot et sur l'Encyclopédie, nº 42, 2007, p. 59-81.

PaUlhan Jean, «La douteuse Justine ou les revanches de la pudeur», dans Les Infortunes de la vertu, Paris, Pauvert, 1959, p. III-XLVI.

SiLver Marie-France, «Le théâtre du marquis de Sade », dans Mieczysław Klimowicz et Aleksander Wit Labuda (dir.), Le Théâtre dans l'Europe des Lumières, Wrocław, Wydawnictwo Uniwersytetu Wrocławskiego, 1985, p. 173-186.

Thomas Chantal, « Juliette, ô Juliette ! (Étude sur la libertine sadienne) », Tel Quel, n 74, 1977, p. 58-67.

— « Isabelle de Bavière : dernière héroïne de Sade », dans Michel Camus et Philippe Roger (dir.), Sade, écrire la crise, Paris, Belfond, 1983, p. 47-66.

— « Rencontres entre Sade et l'univers des pamphlets ou le mal comme unique programme politique », Littérature, no 84, 1991, p. 14-22.

Tourné Maurice, "Pénélope et Circé ou les mythes de la femme dans l'œuvre de Sade », Europe, n 522, 1972, p. 71-88.

Trouille Mary, «The Conflict between Good and Evil, Faith and Irreligion, in Sade's Marquise de Gange ", Eighteenth-century Fiction, vol. 17, n 1,2004, p. 53-86.

\section{Correspondances et études biographiques}

Guilbert Cécile et Leroy Pierre, Cinquante lettres du marquis de Sade à sa femme, éd. Jean-Christophe Abramovici et Patrick Graille, Paris, Flammarion, 2009.

Laborde Alice M., Le Marquis et la Marquise de Sade, Paris, Peter Lang, 1990.

— Le Mariage du marquis de Sade, Paris, Champion, Genève, Slatkine, 1988.

Lely Gilbert, Vie du Marquis de Sade, Paris, Mercure de France, 1952.

Pauvert Jean-Jacques, Sade vivant, Paris, Robert Laffont, 1990.

SADE Marquis de, Lettres à sa femme, éd. Marc Buffat, Arles, Actes Sud, coll. «Babel», 1997. 